\title{
Desarrollo y crisis de las políticas de conciliación de la vida laboral y familiar en España (1997-2014). Un marco explicativo
}

\author{
Inés CAMPILLO POZA \\ Universidad Complutense de Madrid \\ ines.campillo@egeco.es
}

Recibido: 18.07 .2014

Aceptado: 20.01.2015

\section{RESUMEN}

En los últimos años, el análisis de los factores que están en la base del devenir de los Estados del Bienestar postindustriales se ha convertido en una agenda de investigación central en la literatura del bienestar. Los diversos análisis han subrayado el poder explicativo de una o varias de las siguientes hipótesis: la presión de la globalización, el impacto de la Unión Europea, la aparición de "nuevos riesgos sociales" asociados a las transformaciones demográficas y socioeconómicas de las últimas décadas, la movilización de recursos de poder de las izquierdas, y la influencia del diseño políticoinstitucional. Estas hipótesis se han puesto a prueba en los sectores de política social considerados centrales, como las pensiones o la sanidad, pero rara vez han sido examinadas para el caso de las políticas familiares, que paradójicamente constituyen el sector de política social que mayores transformaciones ha experimentado en los últimos años.

El presente artículo aborda este vacío analizando las causas que empujaron el avance de un sector de políticas de conciliación de la vida laboral y familiar en España entre 1997 y 2010 y su posterior declive en los últimos años. Para ello, presenta un modelo "pluralista" de análisis de las transformaciones en las políticas sociales y, en concreto, en la política de conciliación. Frente a la centralidad de las explicaciones institucionalistas en este campo, se recupera la teoría de los recursos de poder y se propone una reformulación en clave feminista. Así, se sostiene que la clave para entender el avance -y posterior declive- de las políticas de conciliación en España es la interacción entre la coyuntura económica y los recursos de poder de las mujeres, sin negar la importancia de otras variables como los nuevos riesgos sociales, la influencia europea y el legado institucional.

Palabras clave: Transformaciones de los Estados del Bienestar. Políticas de conciliación de la vida laboral y familiar. Coyuntura económica. Recursos de poder de las mujeres 


\title{
Development and crisis of reconciliation of work and family life policies in Spain (1997-2014). An explanatory framework
}

\begin{abstract}
In recent years, the analysis of the factors that have pushed the transformation of post-industrial welfare states has become a central research agenda for welfare state scholars. Different analysis of social policies' changes have underlined the explanatory power of one or some of the following factors: the pressure of globalization, the impact of the European Union, the appearance of "new social risks" associated to the socioeconomic and demographic transformations of the last decades, the power resources of leftist organizations, and the influence of the political and institutional arrangements and legacies. Mainstream researchers have usually tested their hypotheses in the sectors of social policy that are considered central, such as pensions or health services. However, these hypotheses have been rarely examined in the case of family policies, which paradoxically constitute the sector of social policy that has suffered the greatest changes in recent years.

The present paper tackles this lacuna through an analysis of the causes that pushed the development and subsequent decline- of reconciliation policies in Spain from 1997 to 2014. To that aim, I present a "pluralist" framework for the study of social policy transformations and, in particular, for the study of reconciliation policy transformations. Against the predominance of institutionalist explanations in this field, I recover the power resources theory and propose a feminist reformulation. Thus, I contend that the key to understanding the development -and subsequent decline- or reconciliation of work and family life policies in Spain is the interaction between the economic situation and women's power resources, factors that in turn interact with other variables such as the new social risks, the influence of the European Union and the institutional legacy.
\end{abstract}

Keywords: Welfare state transformations, reconciliation of work and family life policies, economic situation, women's power resources

\section{INTRODUCCIÓN}

En los últimos veinte años el análisis de los factores que están en la base del devenir de los Estados del Bienestar postindustriales se ha convertido en una agenda de investigación central en la literatura del bienestar. A grandes rasgos, los diversos análisis de las transformaciones de las políticas sociales han subrayado el poder explicativo de una o varias de las siguientes hipótesis: la presión de la globalización (Rieger y Leibfried, 2003; Brady, Beckfield y Seeleib-Kaiser, 2005), el impacto de la Unión Europea (Leibfried y Pierson, 1995; Levy, 1999; Rhodes, 2001; Ferrera y Hemerijck, 2003; Guillén y Álvarez, 2004), la aparición de nuevos riesgos sociales asociados a las transformaciones demográficas y socioeconómicas de las últimas décadas (Adelantado y Gomà, 2000; Esping-Andersen, 2000; Pierson, 2001a; Moreno, 2000; Taylor-Gooby, 2004), la movilización de recursos de poder de las izquierdas (Korpi, 1983; Esping-Andersen, 1993; Huber y Stephens, 2001), y la influencia del diseño político-institucional (Skocpol, 1992; Pierson, 1996, 2001b; Esping-Andersen, 2000; Bonoli, 2001, 2005; Streeck y Thelen, 2005). 
La mayoría de los estudiosos del bienestar ha puesto a prueba estas hipótesis en los sectores de política social considerados centrales, que absorben la mayor parte del gasto social y que presentan una trayectoria más larga, como las pensiones (Myles y Pierson, 2001; Rodriguez Cabrero, 2006; Arza y Kohli, 2008; Ramos y Del Pino, 2009) o la sanidad (Guillén, 2000; Moreno Fuentes, 2009). Sin embargo, raramente han sido examinadas para el caso de las políticas familiares, que paradójicamente constituyen el sector de política social que mayores transformaciones ha sufrido en los últimos treinta años.

Por otro lado, las estudiosas de las políticas familiares, que proceden habitualmente de la corriente feminista de estudio del bienestar, han tendido a ocuparse de la variable dependiente, esto es, se han centrado en qué tipo de políticas públicas se han implementado, en cómo se han implementado, y en los efectos que éstas han tenido en la reorganización social de los cuidados y en la promoción de la (des)igualdad (Valiente, 2000; González, 2004; Fernández y Tobío, 2005; León, 2007; Plantenga y Rémery, 2005; Gornick y Meyers, 2009). No obstante, pocas estudiosas han atendido de forma sistemática a las variables independientes, al porqué de las transformaciones en el área de las políticas de cuidados (como excepción, véanse: Naumann, 2005; Meyer, 2005; Rauch, 2005; Jensen, 2009; Mätzke y Ostner, 2010).

El presente artículo se plantea precisamente abordar este vacío, a través de un análisis de las causas que empujaron el avance de un sector de políticas de conciliación de la vida laboral y familiar en España entre 1997 y $2010^{1}$ y su posterior declive en los últimos años. Resulta de especial relevancia preguntarse por

${ }^{1}$ La acotación del periodo de expansión a 1997-2010 responde a varios motivos. Por un lado, en el ámbito europeo, en 1997 se lanzaba la Estrategia Europea de Empleo, que convertía la conciliación en una de sus áreas de acción preferentes; por otro, en el ámbito doméstico, en ese mismo año se aprobaba el III Plan de Igualdad de Oportunidades entre Mujeres y Hombres (1997-2000), texto normativo que introducía en España la expresión conciliación de la vida laboral y familiar y que preveía ya la puesta en marcha de una ley de conciliación durante su periodo de vigencia. En segundo lugar, en el ámbito europeo, en 2010 se acababa la Agenda 2010 de la Estrategia Europea de Empleo y se ponía en marcha la Estrategia 2020, en la que la conciliación aparecía sólo tangencialmente; en España, en 2010 se agravaba la crisis económica y se introducía el primer paquete de medidas de austeridad, que incluía, entre otras cosas, la puesta en suspenso y la eliminación de algunas de las medidas de conciliación introducidas en el periodo 1997-2009. 
qué la conciliación se convirtió en el cambio de siglo en un sector de política en expansión en nuestro país, caracterizado comúnmente por la literatura del bienestar como un rezagado Estado del Bienestar conservador (Esping-Andersen, 2000) o un Estado familiarista mediterráneo (Moreno, 2000), especialmente cuando no ocurrió lo mismo en el resto de Estados del Bienestar mediterráneos.

Para acometer esta tarea, presento aquí un modelo "pluralista" de análisis de las transformaciones en las políticas sociales, y en concreto en la política de conciliación. Frente a la centralidad de las explicaciones institucionalistas, planteo parafraseando a Evans, Rueschemeyer y Skocpol, 1985- "traer de vuelta el poder" al estudio de los cambios de los Estados del Bienestar postindustriales. Para ello, recupero la teoría de los recursos de poder y propongo una reformulación en clave feminista. De este modo, sostengo que la clave para entender el avance $-\mathrm{y}$ posterior declive- de las políticas de conciliación en España es la interacción entre la coyuntura económica y los recursos de poder de las mujeres, sin negar la importancia de otras variables como los nuevos riesgos sociales, la influencia europea y el legado institucional.

El artículo está estructurado en tres apartados. El primero aborda las características de los análisis habituales de los cambios en las políticas sociales y, en concreto, en las políticas de conciliación de la vida laboral y familiar, y propone, después, un modelo explicativo "pluralista" que recupera una versión modificada de la teoría de los recursos de poder. El segundo apartado describe y analiza la fase de desarrollo de las políticas de conciliación en España de 1997 a 2010 a la luz del modelo presentado. El tercer apartado detalla los recortes que ha sufrido este sector de política desde 2010 y propone una reflexión sobre los problemas y las potencialidades del modelo para dar cuenta de este proceso.

\section{RECUPERANDO EL PODER: LOS RECURSOS DE PODER DE LAS MUJERES}

En los últimos veinte años el estudio de las transformaciones de los programas de bienestar ha estado dominado por el institucionalismo. Si bien existen diversos enfoques institucionalistas, todos comparten un presupuesto teórico básico: las instituciones estatales, una vez consolidadas, son instituciones burocráticas relativamente autónomas, compuestas a su vez por una miríada de instituciones, con reglas, mecanismos, lógicas y personal propios y con una legitimidad social arraigada; por ello, ni son reducibles a mero escenario de lucha de intereses político-económicos enfrentados ni son fácilmente reformables. Los casos de Reino Unido y EE.UU. en los ochenta suelen citarse como ejemplos paradigmáticos de la resistencia de las estructuras fundamentales de los programas de bienestar frente a los embates de la derecha neoliberal más beligerante (Pierson, 1996). 
Si las instituciones estatales, como los programas de provisión social, "tienden a ser cautivos de su propia lógica institucional" (Esping-Andersen, 2000: 14) y tienden, así, a la continuidad, ¿cómo dan cuenta estos enfoques del cambio en los Estados del Bienestar europeos actuales? Por su misma arquitectura argumental, el institucionalismo piensa el cambio en los programas de bienestar como menor y gradual, resultado del ajuste de éstos a nuevos retos o situaciones. Los factores o fuerzas de cambio que suelen enumerarse son básicamente dos: los llamados "nuevos riesgos sociales" y la integración europea. Los primeros hacen referencia a las transformaciones demográficas y socioeconómicas que han sufrido las sociedades capitalistas avanzadas en las últimas cuatro décadas (incorporación masiva de las mujeres al empleo y transformación de los hogares, transformación del mundo del empleo y envejecimiento de la población) y que estarían forzando una redefinición del quién (de los varones sustentadores a las mujeres sustentadoras y los jóvenes) y el qué (de las prestaciones monetarias a los servicios sociales) de la protección social clásica. Más concretamente, se considera que los nuevos riesgos sociales están asociados a las siguientes situaciones: la conciliación de la vida laboral y familiar, la monoparentalidad, los cuidados de los familiares ancianos, la posesión de una baja cualificación o de una formación obsoleta, y una cobertura insuficiente de la seguridad social (Taylor-Gooby, 2004; Bonoli, 2005; Moreno, 2009). La segunda fuente de cambio que mencionan los estudiosos institucionalistas es la integración europea. Frente a quienes sostienen que la Unión Europea estaría promoviendo un proceso de "activación" o "remercantilización" del trabajo en los estados miembros (Serrano y Magnusson, 2007; Papadopoulos, 2005; SeeleibKaiser, 2008), los autores institucionalistas han evaluado las reformas impulsadas por aquélla en términos de "reestructuración" (Pierson, 2001), "re-calibración" (recalibration) (Ferrera y Hemerijck, 2003) o "racionalización" (Guillén y Álvarez, 2004; Guillén y Petmesidou, 2008). La idea central que se esconde tras estas metáforas es que la integración europea no promueve intencionalmente -al menos, hasta ahora- ningún proceso común de signo identificable, sino que únicamente impone un escenario de austeridad que exige a los gobiernos nacionales una reordenación más eficiente de los recursos entre los diversos sectores de política social y entre los diversos tipos de beneficiarios. En todo caso, cualquier reordenación está marcada, sostienen estos autores, por los diversos legados institucionales de los estados miembros, que difícilmente introducirán cambios revolucionarios que redunden en un cambio de régimen.

En el terreno que nos ocupa aquí, cabe subrayar que las pocas autoras que se han preguntado por los factores que han impulsado la introducción o el desarrollo de políticas de conciliación en Europa en los últimos veinte años han tendido a desplegar también una argumentación institucionalista. Las fuerzas de cambio que señalan estas autoras son: el aumento del empleo femenino, el aumento hogares monoparentales y unipersonales, la crisis de los cuidados y la necesidad de 
responder a los objetivos y mandatos europeos. Ante estas presiones de cambio, los diversos Estados responderían de formas diferentes, incorporando el cambio al estilo de política del país. De este modo, estos estudios suelen concentrarse en cómo determinadas recomendaciones o directivas europeas han sido interpretadas, filtradas y modificadas en el ámbito nacional, según el diseño constitucional del país - descentralización o centralización (Rauch, 2005) - y la tradición institucional -por ejemplo: si la atención infantil se ha definido tradicionalmente como política asistencial o como política educativa; si es un país de tradición corporatista, en el que los derechos se entienden asociados al desempeño laboral (León, 2007; Oliver y Mätzke, 2014).

El problema del tipo de explicaciones precedente es que entre la identificación de las fuerzas de cambio y el relato más pormenorizado de la labor de los policy-makers que diseñan una política concreta no parece haber una conexión clara. En primer lugar, algunos riesgos nuevos riesgos sociales tienen ya un recorrido de al menos veinte o treinta años, luego cabe preguntarse por qué son definidos ahora como retos que enfrentar, por qué responden precisamente ahora los gobiernos (Meyer, 2005; MacInnes, 2006; Mätzke y Ostner, 2010). En segundo lugar, incluso aunque las fuerzas de cambio coincidan temporalmente con la respuesta de política pública por parte de los gobiernos, la explicación institucionalista deja sin aclarar el mecanismo causal y los motivos por los que las respuestas difieren según el color del gobierno o entre Estados del Bienestar considerados similares.

Frente a estas explicaciones institucionalistas, a continuación planteo un modelo de análisis que atiende a ese mecanismo causal, interrogándose por el papel del poder en el avance -y crisis- de las políticas de conciliación en nuestro país. Para ello, recupero la teoría de los recursos de poder y propongo una reformulación en clave feminista. De este modo, sostengo que las variables claves para entender el devenir de las políticas de conciliación en España han sido la coyuntura económica y los recursos de poder de las mujeres, que han interaccionado con otras variables como los nuevos riesgos sociales, la influencia europea y el legado institucional.

La teoría de los recursos de poder no es un enfoque novedoso, fue desarrollado durante los años setenta y ochenta para dar cuenta del origen, expansión y consolidación de los Estados de Bienestar de posguerra (Stephens, 1979; Korpi, 1983; Esping-Andersen, 1985; Esping-Andersen y Korpi, 1991; Esping-Andersen, 1993; Wennemo, 1998; Huber y Stephens, 2001). Su formulación clásica rezaría así: en las democracias capitalistas la clase trabajadora es la principal impulsora de los Estados del Bienestar; así pues, donde los recursos de poder de esta clase sean mayores, mayores serán los efectos redistributivos y más universalista será el Estado del Bienestar. Frente al control sobre el capital y los 
medios de producción, los principales recursos de poder de la clase trabajadora son su capacidad organizativa y su grado de influencia política. Estos recursos se medirían a través de siete tipos de indicadores: el nivel de sindicación, el modelo organizativo del movimiento sindical (sindicatos industriales o de oficio; sindicatos unitarios o sindicatos divididos por ideología y/o religión; sindicatos centralizados o descentralizados), el grado de cooperación entre los partidos de izquierdas y el movimiento sindical, el porcentaje de votos recabados por los partidos de izquierdas, el número de puestos del gabinete del gobierno ocupados por partidos de izquierdas, el tiempo que esos partidos han tenido representación en el gobierno, y la fuerza y unión de la oposición política (Korpi, 1983; Esping-Andersen, 1993; Huber y Stephens, 2001).

Con el paso del tiempo, como consecuencia del diálogo con otras perspectivas teóricas, la teoría de los recursos de poder ha sido revisada y modificada en varios aspectos. En primer lugar, ha incorporado ciertos planteamientos de la perspectiva institucionalista de estudios del bienestar. Así, se ha subrayado que los diversos Estados del Bienestar son el producto de la interacción entre las pautas de movilización y coalición de la clase obrera, el legado institucional y la estructura constitucional (Esping-Andersen, 1993; 2000; Huber y Stephens, 2001). En segundo lugar, la revisión más contundente ha sido impulsada por las estudiosas feministas (O'Connor, 1993, 1998; Orloff, 1993; Hobson y Lindhom, 1997; Sainsbury, 1999; Naumann, 2005). Éstas han puesto de manifiesto que la desigualdad social en las democracias capitalistas no sólo es consecuencia de la distribución desigual de recursos económicos, sino también de recursos sociales y culturales; en consecuencia, el conflicto por la redistribución, conflicto constituyente a la par que modelador del Estado del Bienestar, no se restringe a la lucha de clases, incorpora también a otros sujetos, entre los que sobresalen las organizaciones de mujeres (Williams, 2002). Por ello, el devenir de los diversos Estados del Bienestar ni se juega sólo en las arenas políticas tradicionales, ni depende únicamente de los recursos de poder de la clase trabajadora, sino igualmente de los recursos de poder de las mujeres (Hobson y Lindhom, 1997; Mahon, 1997).

De este modo, si los recursos de poder de la clase trabajadora se derivaban de la capacidad organizativa y de la influencia política de los partidos y sindicatos de izquierda, los recursos de poder de las mujeres incluirían también la capacidad organizativa y de la influencia política y discursiva del movimiento de mujeres entendido en un sentido amplio - esto es, como movimiento social autónomo y como organizaciones de mujeres dentro de los partidos políticos, los sindicatos y las administraciones. Los recursos de poder de las mujeres se derivarían, pues, de su doble posición de desigualdad, de clase y de género. Dadas la afinidad política y las tradicionales alianzas o conexiones entre el movimiento de mujeres, los sindicatos y 
los partidos de izquierdas, estos recursos se multiplicarán allá donde gobiernen partidos de izquierda y donde exista un fuerte movimiento sindical. Más concretamente, y en línea con la revisión feminista de esta teoría, los recursos de poder de las mujeres podrían medirse a través de los siguientes indicadores: la visibilidad de las reivindicaciones de las mujeres en la arena discursiva (política, social, mediática); la tasa de empleo femenino; el nivel de sindicación y, especialmente, el nivel de sindicación femenina; el nivel de afiliación a los partidos de izquierda y el nivel de afiliación femenina; la fortaleza de las organizaciones feministas en los partidos, sindicatos y administraciones públicas; la fortaleza y potencial de alianzas del movimiento autónomo feminista; la unidad en términos de reivindicaciones de los partidos de izquierdas, los sindicatos, los organismos de igualdad y el movimiento autónomo feminista; y el tiempo en que los partidos de izquierdas han tenido representación en el gobierno (O'Connor, 1993; Hobson y Lindhom, 1997; Huber y Stephens, 2001; Naumann, 2005).

Una vez identificadas cuáles serían las piezas clave del puzzle según la perspectiva de los recursos de poder de las mujeres, cabe preguntarse cómo encajarían para el caso que nos ocupa. Puesto que partimos de un enfoque "pluralista" y los recursos de poder de las mujeres no son nuestra única variable, deberemos analizar cómo estos recursos han interaccionado a lo largo del periodo de estudio (1997-2014) con el resto de factores que hemos identificado: la situación económica, el impacto de la Unión Europea, los nuevos riesgos sociales y el legado institucional. Como veremos, algunos de estos factores se mantienen relativamente estables durante todos esos años, constituyendo un telón de fondo o una fuerza de cambio constante, mientras que dos de ellos han variado espectacularmente: la coyuntura económica y los recursos de poder de las mujeres. Hemos de esperar, pues, que sean estos dos factores los que puedan arrojar mayor luz sobre los avatares de las políticas de conciliación a lo largo del periodo, que se divide en una primera fase de desarrollo e inversión en conciliación (1997-2010) y en una segunda fase de crisis y retroceso (2010-2014).

\section{DESARROLLO DE LAS POLÍTICAS DE CONCILIACIÓN 1997-2010: BOOM ECONÓMICO, IMPULSO EUROPEO Y RECURSOS DE PODER AMPLIADOS}

Durante el periodo 1997-2010, la problemática de la conciliación de la vida laboral y familiar gana protagonismo en la agenda política española y se convierte en una cuestión central para partidos políticos de diferente color, sindicatos y movimiento feminista. Este protagonismo se traduce en el aumento de iniciativas legislativas que reconocen nuevos derechos o afianzan y amplían los derechos ya existentes. De este modo, el gasto social en familia como porcentaje del PIB pasa del 0,41\% en 1995 al 1,51 en 2009 (OCDE), el gasto en cuidados a ancianos pasa 
del $0,16 \%$ al $0,45 \%$ en 2008 (Eurostat), mientras que la tasa neta de escolarización de 0 a 2 años pasa del 5,5\% en 1995 al 28,6\% en 2010 (Ministerio de Educación, Cultura y Deporte). Este desarrollo no sólo diferencia a España del resto de regímenes familiaristas del sur de Europa, con los que normalmente se la agrupa, sino que ha llevado a algunas investigadoras a preguntarse por una posible ruptura de su tradicional trayectoria institucional (Campillo, 2013; León y Migliavacca, 2013). La pregunta es, pues: ¿por qué ocurre esto en nuestro país?, ¿cuáles han sido las fuerzas de cambio?

Como he señalado en el punto anterior, los trabajos que han abordado el desarrollo de este tipo de políticas en este periodo han señalado, implícita o explícitamente, que el avance ha estado motivado por ciertas transformaciones sociodemográficas y por los imperativos europeos. Sin negar la importancia de estas fuerzas de cambio, aquí sostengo que han actuado como ventanas de oportunidad, pero no son condiciones suficientes para explicar el proceso. Una prueba de ello lo constituyen los casos de otros países europeos igualmente familiaristas, como Italia o Grecia, que no han experimentado un impulso particular de este tipo de políticas en los últimos años, pese a estar igualmente sometidos a esas fuerzas de cambio. Como veremos a continuación, los principales determinantes del desarrollo de políticas de conciliación en España en el periodo 1997-2010 han sido el espectacular crecimiento económico y la creciente movilización de recursos de poder de las mujeres desde mediados de los noventa, cuando aumentan significativamente tanto la tasa de empleo femenino, como la tasa de afiliación femenina a partidos y sindicatos, y cuando el feminismo -entendido en un sentido amplio- recupera parte de la visibilidad y aceptación social y mediática que había tenido durante la transición democrática. Esta multiplicación de los recursos de poder de las mujeres se traduce en una influencia directa e indirecta sobre los diferentes gobiernos a lo largo del periodo, fueran conservadores (19962004) o socialdemócratas (2004-2010). No obstante, dadas la afinidad política y las tradicionales conexiones entre el movimiento de mujeres, los sindicatos y los partidos de izquierdas, esa influencia es mayor, más directa y evidente, sobre los gobiernos del Partido Socialista (2004-2010), que no sólo ponen en marcha un mayor número de medidas, sino que las dotan de un carácter -al menos pretendidamente- más igualitario. Veamos más detenidamente cómo han interactuado los factores mencionados en esas dos etapas.

Tras catorce años de gobiernos socialistas, el Partido Popular gana las elecciones en 1996 y se mantiene en el poder hasta 2004. Su acceso al gobierno no supuso la fractura esperada en el feminismo institucional y en las políticas de igualdad (Valiente, 1996; Bustelo y Ortbals, 2007). Los gobiernos conservadores mantuvieron el Instituto de la Mujer y pusieron en marcha, primero, el III Plan de Igualdad de Oportunidades entre Hombres y Mujeres (1997-2000) y, más tarde, el 
IV PIOHM (2003-2006). En el campo de la conciliación, la primera actuación del gobierno del PP fue la aprobación del Real Decreto-Ley 15/1998 "de medidas urgentes para la mejora del mercado de trabajo en relación con el trabajo a tiempo parcial y el fomento de su estabilidad". De acuerdo con lo establecido por la directiva europea relativa al trabajo a tiempo parcial $(97 / 81 / \mathrm{CE})$, se regulaba este tipo de trabajo, garantizando la igualdad de trato y no discriminación de los trabajadores a tiempo parcial en relación con los trabajadores a tiempo completo, regulando su derecho a la protección social, y promoviendo la movilidad voluntaria de un trabajo a tiempo completo a uno a tiempo parcial o viceversa (RD-Ley 15/1998).

En segundo lugar, tal y como preveía el III Plan de Igualdad, el gobierno de PP aprueba en 1999 la ley de "conciliación de la vida familiar y laboral de las personas trabajadoras" (Ley 39/1999). Pese a que la exposición de motivos identifica la desigualdad de género como el problema clave a combatir, el cuerpo de la ley y los derechos que ésta reconoce están fundamentalmente dirigidos a las madres trabajadoras ${ }^{2}$. Básicamente, el texto recoge e integra los derechos de conciliación previamente existentes, pero dispersos en diversas normativas, y los amplía no sólo temporalmente, sino también con respecto a quién y en qué casos pueden ser disfrutados. De este modo, la ley amplía, para hombres y mujeres, los casos en los que es posible una reducción de jornada con reducción proporcional de la retribución (para el cuidado de hijos prematuros, familiares dependientes o con discapacidad hasta el segundo grado de consanguinidad), reconoce el permiso retribuido de dos días para los casos de adopción y enfermedad grave de un familiar, amplía a diez semanas el periodo del permiso de maternidad que la madre puede ceder al padre, e iguala la duración del permiso de maternidad en caso de adopción o acogimiento. Ahora bien, la ley de conciliación crea también nuevos derechos, asociados directamente a las recomendaciones de las directivas 92/85/CEE y 96/34/CE: en primer lugar, establece un nuevo permiso retribuido para la realización de las técnicas de preparación al parto, y reconoce la suspensión del contrato por riesgo durante el embarazo; en segundo lugar, contempla que el permiso de maternidad pueda ser disfrutado a tiempo parcial; en tercer lugar, reconoce el derecho a excedencias también para el cuidado de familiares dependientes (hasta un año); por último, establece bonificaciones en las contribuciones a la Seguridad Social para aquellas empresas que sustituyan a los trabajadores que disfruten de algún permiso para el cuidado.

${ }^{2}$ De este modo, como señalan Bustelo y Peterson (2005), el problema deja de ser un problema social para ser un problema de las mujeres trabajadoras, que -parece considerarse- debieran acercarse a la "norma" masculina de empleo. Para un excelente análisis pormenorizado de los discursos de ésta y otras normas de conciliación, véase: Peterson, 2007, 2011. 
Aunque el III PIOHM preveía el impulso de los servicios dirigidos a los menores de 0 a 3 años, ni la ley de conciliación ni ningún otro tipo de actuación aborda la promoción de este tipo de servicios entre 1996 y 2000. Más bien al contrario, en 1998 el gobierno popular aprueba la Ley de Reforma del IRPF (Ley 40/1998), que suprime la deducción fiscal por gastos de guardería introducida en 1991 por el gobierno socialista. No obstante, apenas dos meses antes de las elecciones generales de marzo de 2000, el gobierno aprueba unas "Medidas de mejora de la protección familiar de la Seguridad Social" (Ley 1/2000), que incluían una prestación única, sometida a comprobación de recursos, por nacimiento del tercer hijo $-\mathrm{y}$ siguientes- $\mathrm{y}$ ayudas monetarias por parto múltiple.

Durante su segunda legislatura (2000-2004), el gobierno conservador pone en marcha el Plan Integral de Apoyo a la Familia (2001-2004). Éste se proponía promover el igual acceso de hombres y mujeres al mercado de trabajo y la extensión de los servicios de atención a la primera infancia, en colaboración con las Comunidades Autónomas y los municipios; no obstante, finalmente queda reducido a la creación de una deducción fiscal de cien euros mensuales para las madres trabajadoras con hijos menores de tres años (Ley 46/2002). La extensión de la oferta de plazas públicas de educación infantil queda de nuevo sin cumplir y una nueva ley educativa retira del sistema educativo el ciclo de 0 a 3 años, que se convierte en un ciclo preescolar de carácter asistencial, sin objetivos ni requisitos mínimos, salvo los que fijara voluntariamente cada Comunidad Autónoma (LOCE, Ley 10/2002).

Aunque pueda resultar paradójico que sea precisamente un gobierno conservador quien introduzca definitivamente la problemática de la conciliación en la agenda política española, este hecho debe comprenderse a la luz de una serie de circunstancias coincidentes: en primer lugar, el legado del feminismo institucionalizado a lo largo de los años ochenta y noventa, cristalizado no sólo en ciertas estructuras del Estado -como el Instituto de la Mujer-, sino también en los estatutos y aparatos de los partidos y sindicatos (secretarías de la mujer o de igualdad; sistemas de cuotas en los órganos de dirección), en las estructuras y programas académicos, y en el mundo de la consultoría y la investigación; en segundo lugar, el despegue económico y el crecimiento constante del empleo que vive nuestro país a partir de 1995, tras la crisis de principios de los noventa; en tercer lugar, la necesidad de cumplir con los requisitos europeos, trasponiendo las directivas sobre protección de la maternidad (92/85/CEE), permisos parentales (96/34/CE) y promoción del tiempo parcial (97/81/CE), y asumiendo el programa de activación de la Estrategia Europea de Empleo; en cuarto lugar, la voluntad de revitalizar las cuestiones relacionadas con la familia, ausentes de la agenda política democrática desde la transición (León, 2011); por último, la creciente movilización de recursos de las mujeres, derivados del aumento del empleo femenino y de la 
mayor presencia de las mujeres en la vida pública: el empleo femenino, que se había reducido entre 1975 y 1985, vuelve a recuperarse a principios de los años noventa y aumenta significativamente entre 1995 y 2000 (véase Gráfico 2.1). Pero la multiplicación de los recursos de las mujeres se plasma también en el aumento de la afiliación femenina en partidos y sindicatos (véase Gráfico 2.1) y en la reactivación del movimiento social feminista ${ }^{3}$. María Martínez ha tratado de cuantificar a grandes rasgos este resurgir feminista, para lo que se ha servido de dos estudios del Instituto de la Mujer sobre el asociacionismo de mujeres que estimaban que los grupos de mujeres de ámbito estatal habrían pasado de 58 en 1989 a 186 en 1997 (Martínez, 2011: 16-17). Pese a que estos datos han de ser tomados con precaución, puesto que no todas las asociaciones de mujeres son asociaciones feministas, creo que dan una idea aproximada de la activación del movimiento feminista desde los años noventa. Los resultados para España de la Word Social Survey sobre pertenencia a grupos de mujeres, que pasa del $0,8 \%$ de las entrevistadas en 1990 al 1,7 en 2000, parecen apuntar también a esta tendencia (World Social Survey). Otro indicador del aumento de los recursos de las mujeres en este periodo es el cambio que comienza a experimentar la representación pública del feminismo: la complacencia social y mediática con la igualdad formal conquistada por las mujeres durante la transición y los ochenta da paso a la consciencia de la persistente desigualdad real y, así, al "redescubrimiento" de la utilidad de la lucha feminista. Este cambio en la representación social del feminismo en España está relacionado, en gran medida, con la visibilidad que el fenómeno de la violencia doméstica empieza a ganar en los medios de comunicación entre 1997 y 1998, a raíz de una oleada de casos sangrantes de asesinatos de mujeres a manos de sus compañeros y de la negligencia o machismo judicial asociada a esos casos (Boix, 2001). Pese a que el movimiento feminista venía trabajando el tema de la violencia machista desde hacía años, aquellos hechos desencadenan una serie de manifestaciones y actos públicos que ponen en el centro de la agenda política la necesidad de tomar medidas urgentes e integrales al respecto (Valiente, 2005: 105).

${ }^{3}$ En 2000 la celebración de la Marcha Mundial de las Mujeres estimula la movilización feminista en muchas regiones de España, y la Federación de Organizaciones Feministas del Estado Español aprovecha esa fecha para organizar las Jornadas Feministas Estatales "Feminismo es... y será" en Córdoba, a las que acuden unas 3.000 asistentes (El País, 08/12/2000), entre ellas muchas jóvenes, lo que sorprende a las propias organizadoras (Martínez, 2011: 14)

${ }^{4}$ De hecho, el gobierno conservador se ve forzado a aprobar un Plan de Acción Contra la Violencia Doméstica en 1998, en el marco del cual se modifica el Código Penal y la ley de enjuiciamiento criminal con el fin de incluir el delito de "violencia psíquica ejercida con carácter habitual" y una "nueva medida cautelar que permita el distanciamiento físico entre el agresor y la víctima" (Ley 14/1999). 
En marzo de 2004 se produce en España un cambio de gobierno, lo que supone en cierto modo un cambio de fase en el desarrollo de las políticas de conciliación de la vida laboral y familiar. En particular, la primera legislatura del gobierno de Zapatero (2004-2008) está marcada por el compromiso con la igualdad de género y la conciliación, que se traduce en la introducción de una variedad de medidas. En primer lugar, una nueva ley de educación, la Ley Orgánica de Educación (LOE, Ley 2/2006), reintegró los servicios de atención infantil de 0 a 3 años en el sistema educativo, pero sin estipular los mínimos y garantías debían cumplir. Además, la Ministra de Educación y Política Social, Mercedes Cabrera, presentó durante el verano del 2008 el Plan Educa3, que preveía una inversión de 1.087 millones de euros entre 2008 y 2012 para impulsar la creación de nuevas plazas educativas para menores de 3 años, plazas que cifraba en 300.000 (El País, 30 agosto 2008). Sin embargo, el agravamiento de la crisis a partir de 2009-2010 y la disminución concomitante de las aportaciones de las Comunidades Autónomas frustró los objetivos de este plan, que poco antes de las elecciones de 2011 sólo había conseguido crear 71.000 plazas, esto es, el 23\% de las plazas prometidas para 2012 (El País, 25 agosto 2011).

En segundo lugar, el "Plan Concilia", acordado entre el gobierno y los sindicatos en la Mesa General de Negociación, y dirigido a los trabajadores y trabajadoras de la Administración General del Estado, entró en vigor en enero de 2006. Este plan reconoce para este tipo de trabajadores y trabajadoras, entre otras medidas, un permiso de paternidad de 10 días, la sustitución del permiso de lactancia por una ampliación adicional de cuatro semanas del permiso de maternidad, una reducción de la parte fija y flexible del horario, la posibilidad de una reducción y/o flexibilización de jornada para quienes tengan a su cuidado hijos menores de 12 años y la posibilidad de una reducción del $50 \%$ de la jornada durante un mes, con carácter retribuido, para atender al cuidado de un familiar en primer grado por razón de enfermedad grave.

En tercer lugar, en diciembre de 2006, el gobierno socialista aprobó la ley de "Promoción de la Autonomía Personal y Atención a las personas en situación de dependencia" (Ley 39/2006). En su exposición de motivos, la ley reconoce que el cuidado de las personas dependientes ha recaído tradicionalmente en las mujeres, sin embargo, constata que los actuales cambios en el modelo de familia y la incorporación masiva de las mujeres al mercado de trabajo hacen imprescindible el compromiso público para con el cuidado de aquellas personas. La ley establece que todas las personas dependientes - definidas como personas mayores incapaces de valerse por sí mismas o personas con discapacidad- tienen derecho a ser atendidas, según su renta y su grado de dependencia, en tres modalidades: por los servicios públicos o concertados de atención y cuidado, recibiendo una prestación económica, para comprar esos servicios de cuidado en el mercado o, en su defecto, 
recibiendo una prestación económica para cuidados en el entorno familiar. El desarrollo de esta ley, que se puso en marcha a finales de 2007, se vio truncado desde su mismo comienzo. Como apuntó certeramente Rodríguez Cabrero en 2009, desde su creación el Sistema para la Autonomía y la Atención a la Dependencia (SAAD) se ha encontrado con diversos problemas: la descoordinación entre las administraciones implicadas -Estado central, Comunidades Autónomas y ayuntamientos- en el intercambio de información y en los ritmos de aplicación; el desbordamiento de las previsiones relacionadas con la población objeto de derecho; y la perversión de la propia ley, cuyo texto priorizaba la inversión en servicios y cuya puesta en práctica ha favorecido las prestaciones económicas, especialmente la prestación "para cuidados en el entorno familiar" (El Pais, 25/02/2009). Estos problemas se han traducido en una demora generalizada tanto en la valoración de las solicitudes como en el cobro de las prestaciones y, hasta 2010, en una inyección suplementaria de fondos para el correcto desarrollo de la ley ${ }^{6}$. A estas complicaciones se han sumado, desde 2010, los sucesivos recortes presupuestarios, que han puesto en suspenso la viabilidad del SAAD.

En cuarto lugar, en marzo de 2007, el gobierno socialista aprobó ley "para la igualdad efectiva de mujeres y hombres" (Ley 3/2007), ley transversal que afecta a diversos terrenos y políticas públicas, y que se propone, entre otras cosas, fomentar una mayor corresponsabilidad entre mujeres y hombres en la asunción de las obligaciones familiares. Para ello, la ley regula la creación de un permiso exclusivo para los padres de 13 días, a los que se suman los 2 días por nacimiento de hijo que ya reconocía el Estatuto de los Trabajadores ${ }^{7}$. Asimismo, la ley debilita el vínculo entre el derecho a la baja de maternidad y el desempeño laboral, al reducir los días cotizados que son necesarios para generar tal derecho y crear un

${ }^{5}$ El texto de la ley calificaba de "excepcional" la ayuda económica "para cuidados en el entorno familiar". Sin embargo, frente a la escasez de oferta pública de centros y servicios públicos de cuidados, se ha recurrido de forma generalizada a esta ayuda, lo que tiene implicaciones serias en términos de igualdad de género. La generalización de esta ayuda significa la legitimación y perpetuación de aquello que la ley pretendía combatir: la asunción por parte de las mujeres del trabajo de cuidados y la tradicional división sexual del trabajo en el seno de las familias.

${ }^{6}$ Desde que la ley entró en vigor hasta 2010-2011, los Presupuestos Generales hubieron de dedicar una cuantía mayor de la que proyectaba la memoria económica inicial de la ley. En 2008 se dedicaron 871 millones de euros para impulsar el Sistema para la Atención a la Dependencia (MEH, 2007), en 2009, 1.159 millones de euros, y en 2010, 1.581 millones de euros (El País, 30/9/2009).

${ }^{7}$ Es importante subrayar que en junio de 2009 el Congreso de los Diputados aprobaba por unanimidad la proposición de ley, presentada por CIU, para la ampliación a un mes del permiso de paternidad. Ésta debía entrar en vigor en enero de 2011, pero los Presupuestos de 2011 aprobados por el gobierno de Zapatero anularon su puesta en marcha. 
subsidio no contributivo de maternidad de seis semanas para las mujeres que no cumplen el periodo mínimo de cotización estipulado.

Por último, el gobierno socialista introdujo, meses antes de las elecciones de marzo de 2008, el polémico "cheque bebé", una prestación única de 2500 euros por nacimiento o adopción de hijo, que las madres podían deducir en su declaración de la renta o cobrar como prestación no contributiva de la seguridad social (Ley $35 / 2007)$.

El aumento en el número y en la variedad de instrumentos de conciliación que se ponen en marcha entre 2004 y 2008 ha de entenderse a la luz de la intensificación de algunos de los factores que mencionaba para la etapa anterior: en primer lugar, un contexto propicio de fuerte crecimiento económico, marcado por la expansión del crédito, la burbuja inmobiliaria, un crecimiento del empleo continuado, especialmente del empleo femenino, y superávits presupuestarios; en segundo lugar, la voluntad de adecuarse a las recomendaciones de la Estrategia Europea de Empleo, que en 2002 había adoptado los objetivos de una cobertura mínima de la educación infantil de 0 a 3 años del $33 \%$ y de 3 a la edad de escolarización del 90\%; por último, la multiplicación de los recursos de poder de las mujeres. La teoría de los recursos de poder toma la tasa de empleo femenino como el principal indicador o termómetro de los recursos de poder de las mujeres (Huber y Stephens, 2001). La idea que se esconde tras ello es que una mayor tasa de empleo femenino implica indirectamente una mayor influencia de las mujeres sobre la agenda política, a través de su mayor participación en sindicatos y en partidos políticos de izquierda. Esto mismo ocurre en España en esta etapa, como podemos ver en el Gráfico 2.1. Es más, no sólo aumenta la participación de las mujeres en partidos y sindicatos, sino también la movilización feminista. Martínez menciona a este respecto que el registro de asociaciones de mujeres del Instituto de la Mujer de Andalucía computaba 367 asociaciones en esa comunidad en 1997 y más de 1500 en la actualidad (Martínez, 2011: 16-17). En la misma línea, la World Social Survey muestra un cambio en la confianza en el movimiento de mujeres en España, que pasa de despertar el 43,6\% de "bastante o mucha" confianza en 1995 al 58,4\% en 2007 (Word Social Survey). 
Gráfico 2.1. Evolución de la tasa de actividad femenina (TAF) y de la ratio femenina sobre el total de la afiliación a los principales partidos políticos y sindicatos

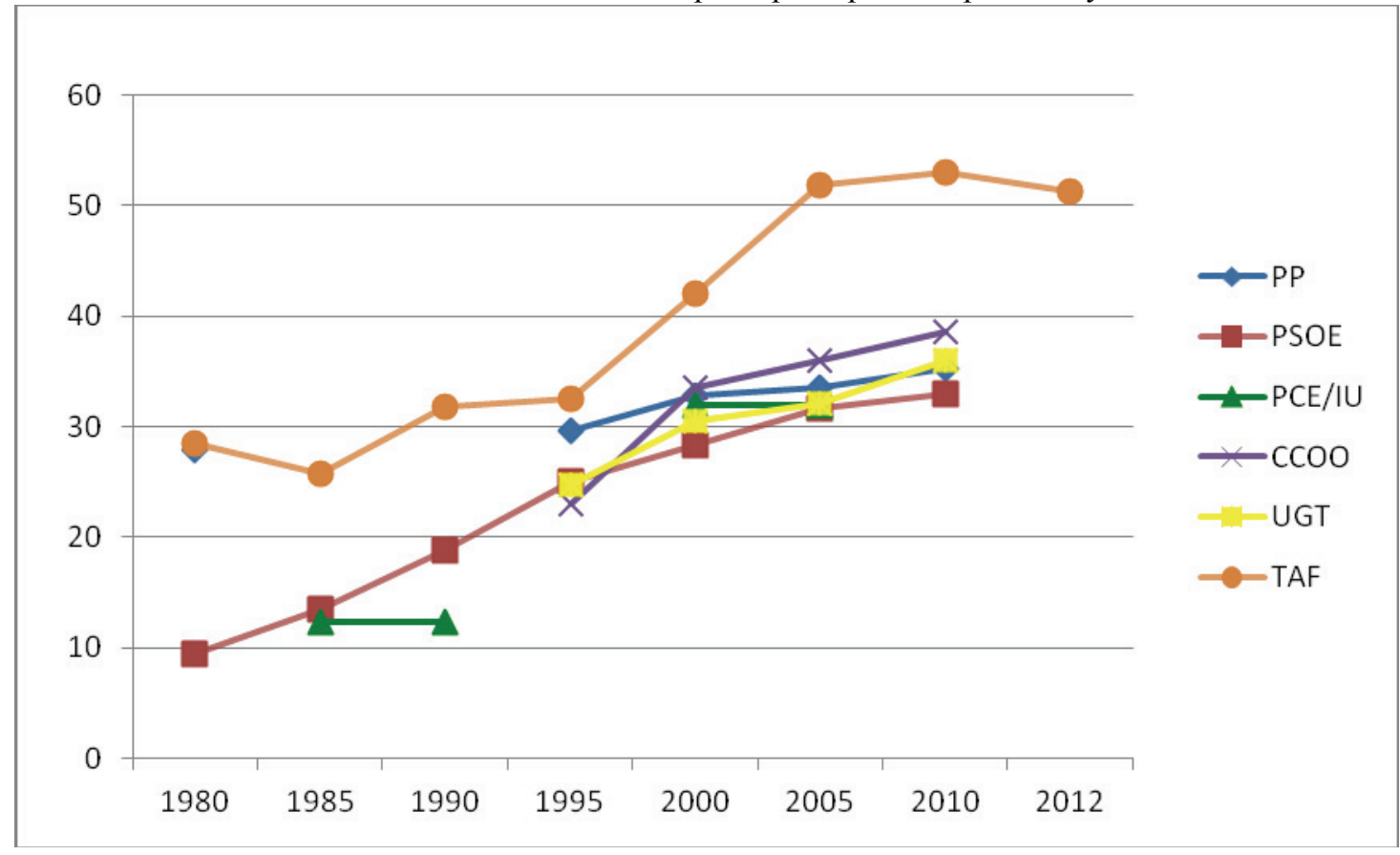

Fuente: Elaboración propia, a partir de OCDE, Instituto de la Mujer y Verge (2006).

No obstante, cuando en marzo de 2008 Zapatero vuelve a ganar las elecciones generales, el contexto es completamente distinto: la crisis económica mundial, caracterizada tanto por la subida del precio del petróleo y las materias primas, como por la crisis crediticia e hipotecaria y la bancarrota de diversas entidades financieras, azota especialmente las economías de Estados Unidos, Irlanda o España. Así pues, la segunda legislatura del Partido Socialista (20082011) va a estar profundamente marcada por la recesión económica, el desempleo creciente y el déficit presupuestario. No obstante, la inversión en políticas de conciliación -y política social en general- sigue aumentando hasta 2010 , cuando se introducen los primeros recortes sociales.

\section{CRISIS (2010-2014): CRISIS ECONÓMICA, PÉRDIDA DE IMPULSO EUROPEO Y RECURSOS DE PODER REDUCIDOS}

2010 marca un hito en la evolución de las políticas de conciliación en Europa y, especialmente, en España. En el ámbito europeo, se acaba la Agenda 2010 de la Estrategia Europea de Empleo y se pone en marcha la Estrategia 2020, 
una estrategia marcadamente activadora, en la que desaparecen los objetivos de política de igualdad de oportunidades y de conciliación. En nuestro país, en 2010 se agrava la crisis económica, la tasa de desempleo alcanza el $20 \%$ y se intensifica la presión de Bruselas. En mayo, el gobierno socialista aprueba el primer paquete de medidas de austeridad, que incluye, entre otros recortes sociales (recorte de sueldos públicos, congelación de las pensiones), la puesta en suspenso y la supresión de algunas de las medidas de conciliación introducidas en el periodo 1997-2009: la eliminación del "cheque-bebé", un recorte del 5,2\% en el presupuesto para la dependencia y la cancelación de la ampliación del permiso de paternidad a un mes. Asimismo, a lo largo de 2010 y 2011, se aprueban tres reformas tremendamente regresivas: la reforma del mercado de trabajo, que generaliza el contrato de fomento del empleo (con menor indemnización por despido), introduce la crisis de la empresa como causa objetiva para el despido procedente y permite la salida de los convenios laborales vigentes; la reforma de las pensiones, que aumenta el periodo de cotización necesario para generar pensión, aumenta la edad de jubilación a los 67 años e incrementa también el periodo cálculo de la pensión de los últimos 15 años trabajados a los últimos 25; y, por último, la reforma -pactada con el PP- del artículo 135 de la Constitución, que blinda constitucionalmente la estabilidad presupuestaria. Todas estas medidas sellarán la pérdida de legitimidad del gobierno socialista y el divorcio entre gobierno y sindicatos (que convocan una huelga general el 29 septiembre de 2010), y forzarán el adelanto de las elecciones.

En noviembre de 2011, el Partido Popular gana las elecciones con mayoría absoluta. Desde entonces, el gobierno conservador ha ahondado en la agenda de austeridad iniciada por el gobierno socialista. La cantidad y el calado de las reformas y recortes sociales aprobados hasta el momento ha sido tal, que resulta difícil dar cuenta aquí de todas ellas. Entre los recortes introducidos cabe, sin duda, destacar: la congelación de los sueldos y la prolongación de la jornada laboral de los empleados públicos; la congelación de la creación de empleo público, excepto en fuerzas de seguridad del Estado, sanidad y educación, que admiten un $10 \%$ de reposición; la reducción de las cuantías de la prestación y los subsidios por desempleo; el aumento del control sobre los beneficiarios de prestaciones y subsidios de desempleo; y la supresión de la universalidad de la sanidad pública, con la introducción del copago para medicamentos y otros productos sanitarios, y con la retirada de la tarjeta sanitaria a inmigrantes sin papeles. Asimismo, el gobierno conservador aprueba, en febrero de 2012, una segunda reforma del mercado laboral y, en noviembre de 2013, una segunda reforma de las pensiones. La primera reduce los costes de despido de los contratos indefinidos y amplía las causas de despido objetivo, limita la vigencia de los convenios colectivos vencidos, permite el descuelgue de los convenios, da prioridad a los convenios de empresa frente a los convenios de ámbito superior y permite al empresario modificar las condiciones de trabajo de sus trabajadores -entre ellas, el salario, la jornada, el 
horario y la distribución del tiempo de trabajo, permitiendo horas extraordinarias en contratos a tiempo parcial. Esta reforma motiva una huelga general el 29 de marzo de 2012, la segunda de este cuatrienio. Por su parte, la nueva reforma de las pensiones desliga la actualización de las pensiones de la inflación e introduce un factor de sostenibilidad que vincula la cuantía con la esperanza de vida.

Más allá de los efectos que las reformas y recortes mencionados tienen en la (des)igualdad de género y en la conciliación, se calcula que en los dos primeros años del gobierno conservador se ha recortado un $47 \%$ en políticas de igualdad y un $30 \%$ en políticas de prevención de la violencia contra las mujeres (Público, 20/11/2013). En cuanto al SAAD, como declaraba el Presidente de la Asociación Estatal de Directoras y Gerentes de Servicios Sociales ante la Subcomisión para el Estudio de la Racionalización de los Horarios y la Conciliación del Congreso, se han recortado progresivamente derechos y presupuestos, lo que supone "demoler controladamente la ley" (Cortes Generales, 2013: 30): la prestación económica para cuidados en el entorno familiar se ha reducido entre un $15 \%$ y un $30 \%$, se ha eliminado la cotización a la Seguridad Social de los cuidadores y cuidadoras no profesionales, se ha aplazado la incorporación al sistema de los dependientes moderados, se han incrementado las aportaciones de los usuarios, se ha ampliado el plazo suspensivo para el posible pago retroactivo de las ayudas una vez concedidas y se ha eliminado también la compatibilidad entre servicios. Por otro lado, Unicef ha estimado que la inversión en políticas de infancia se ha reducido un $14,6 \%$ entre 2010 y 2013 (UNICEF, 2014: 32).

¿Cómo ha sido posible tal ataque a las políticas de conciliación y, en general, a las políticas sociales? Decíamos que el desarrollo de estas políticas entre 1997 y 2010, y especialmente entre 2004 y 2008, había estado motivado por una serie de factores: las transformaciones demográficas y socioeconómicas de los últimos treinta años; el feminismo institucionalizado durante los años ochenta y noventa; la influencia de la agenda de conciliación de la Unión Europea, primero, a través de directivas y, más tarde, a través de la Estrategia Europea de Empleo; el boom económico, marcado por la burbuja inmobiliaria, la expansión del crédito, superávits presupuestarios continuados y un espectacular crecimiento del empleo; y la multiplicación de los recursos de poder de las mujeres derivada del aumento del empleo femenino y de la mayor presencia de éstas en la esfera pública. ¿Qué cambia entre 2008-2010 y 2014? Cambia, principalmente y de forma drástica, la situación económica: esta etapa está marcada por la recesión económica, el déficit presupuestario y un intenso crecimiento del desempleo, que pasa del $11,34 \%$ en 2008 al 26, 36\% en 2013 (INE). La crisis económica y el espectacular aumento del desempleo se traducen, a su vez, en un debilitamiento de los recursos de poder de las mujeres. Por un lado, las debilita como trabajadoras, al condenarlas al desempleo o a la precariedad, empujándolas a reducir su participación en partidos, 
sindicatos u otro tipo de organizaciones; por otro, debilita también su posición en la esfera privada, al aumentar las necesidades que ya no pueden cubrir pagándolas en el mercado y que tampoco cubre un Estado del Bienestar recortado. Esta reducción de los recursos de poder de las mujeres queda patente, como vemos, en la evolución paralela que ha sufrido en los últimos años la tasa de empleo femenino y la afiliación a los partidos de izquierda y a los sindicatos (véase Gráfico 2.1). Así, las políticas de conciliación de la vida laboral y familiar, que parecían ser una prioridad para partidos, sindicatos y policy-makers en la época de expansión, han desaparecido rápidamente de la agenda política.

\section{CONCLUSIÓN}

El objetivo de este artículo era explorar los determinantes del desarrollo de las políticas de conciliación de la vida laboral y familiar en España entre 1997 y 2010 y su posterior declive. Para ello, he presentado un marco "pluralista" de estudio de las transformaciones de política social que toma en consideración las diversas variables identificadas en los estudios convencionales (cambios sociodemográficos, U.E. y legados institucionales), al tiempo que incluye dos variables poco consideradas habitualmente: la coyuntura económica y los recursos de poder. De hecho, he sostenido que los factores explicativos clave del avance $-\mathrm{y}$ posterior declive- de las políticas de conciliación en España han sido principalmente la interacción entre la coyuntura económica y los recursos de poder de las mujeres.

No obstante, a la luz de los acontecimientos de los últimos años, cabe hacerse la siguiente pregunta: si los avances en conciliación del periodo 1997-2010 han quedado reducidos a cenizas en tan poco tiempo, ¿cuán sólida es la hipótesis de los recursos de poder de las mujeres incluso para dar cuenta del periodo de expansión de esas políticas? Aunque, sin duda, la respuesta a esta pregunta merece mayor investigación y quizá mayor distancia temporal, creo que los actuales acontecimientos no invalidan el modelo que he presentado aquí. Puesto que la influencia europea, el peso del legado institucional y la presión de los cambios sociodemográficos existían anteriormente a este periodo y siguen existiendo en la actualidad, el descarte de la hipótesis de los recursos de poder nos llevaría a señalar al crecimiento económico y la mayor disponibilidad de recursos públicos como factor clave del desarrollo de las políticas de conciliación. Sin embargo, esta explicación dejaría sin aclarar, por un lado, el mecanismo por el que esa mayor disponibilidad de recursos se tradujo en una mayor inversión en conciliación y, por otro, las diferencias en cantidad e intensidad de las medidas de conciliación puestas en marcha por los distintos gobiernos. La hipótesis de los recursos de poder de las mujeres nos permite entender mejor el mecanismo que une situación económica con avance $-\mathrm{o}$ retroceso- de las políticas de conciliación y nos da pistas sobre las 
diferencias a este respecto entre los gobiernos conservadores y los gobiernos socialdemócratas.

Sin embargo, la velocidad con la que se han eliminado o desvirtuado muchas de las medidas de conciliación introducidas durante el periodo de expansión (1997-2010) nos sugiere que, probablemente, para que un sector de política se mantenga en el tiempo, no sólo es necesario que se multipliquen los recursos de poder de las mujeres, sino que se consoliden durante un periodo más largo de tiempo, cristalizándose en dinámicas e inercias institucionales más difícilmente reformables.

\section{BIBLIOGRAFÍA}

ADELANTADO, JOSÉ y GOMÀ, RICARD (2000): "El contexto: La reestructuración de los regímenes de bienestar europeos" en J. Adelantado (Ed.), Cambios en el Estado del Bienestar. Políticas sociales y desigualdades en España, Barcelona: Icaria: 63-96.

ARZA, CAMILA y KOHLI, MARTIN (Ed.) (2008): Pension Reform in Europe. Politics, policies and outcomes, Abingdon: Routledge.

BOIX, MONTSERRAT (2001): "El tratamiento de la violencia hacia las mujeres en los medios de comunicación", I Congreso Nacional sobre la Violencia contra la Mujer, Gijón, 11, 12 y 13 octubre.

BONOLI, GIULIANO (2001): "Political Institutions, Veto Points, and the Process of Welfare State Adaptation" en P. Pierson (Ed.), The New Politics of the Welfare State, New York: Oxford University Press: 238-64.

BONOLI, GIULIANO (2005): "The politics of the new social policies: providing coverage against new social risks in mature welfare states", Policy \& Politics, 33 (3): 432-49.

BRADY, DAVID, BECKFIELD, JASON y SEELEIB-KAISER, MARTIN (2005): "Economic Globalization and the Welfare State in Affluent Democracies, 19752001", American Sociological Review, 70: 921-48.

BUSTELO, MARÍA y ORTBALS, CANDICE D. (2007): "The Evolution of Spanish State Feminism: a Fragmented Landscape" en J. Outshoorn y J. Kantola (Ed.), Changing State Feminism, Basingstoke: Palgrave Macmillan: 201-23.

BUSTELO, MARÍA y PETERSON, ELIN (2005): "Conciliación y (des)igualdad. Una mirada debajo de la alfombra de las políticas de igualdad entre mujeres y hombres", Somos, 7.

CAMPILLO, INÉS (2010): "Políticas de conciliación de la vida laboral y familiar en los regímenes de bienestar mediterráneos: los casos de Italia y España", Política y Sociedad, 47 (1): 189-213.

CAMPILLO, INÉS (2013): ¿Adiós al familiarismo? Las políticas de conciliación de la vida laboral y familiar en España, 1997-2010, Tesis doctoral Universidad Complutense de Madrid, Madrid. 
CORTES GENERALES (2013): Subcomisión para el estudio de la Racionalización de Horarios, la Conciliación de la Vida Personal, Familiar y Laboral y la Corresponsabilidad. Informe de la Subcomisión. 26 de septiembre de 2013, núm. 330 .

DEL PINO, ELOÍSA (2009): "Un marco conceptual para estudiar el cambio del Estado del Bienestar y las políticas sociales" en L. Moreno (Ed.), Reformas de las políticas del bienestar en España, Madrid: Siglo XXI: 35-66.

ESPING-ANDERSEN, GOSTA (1993): Los tres mundos del Estado del Bienestar, Valencia: Alfons el Magnànim.

ESPING-ANDERSEN, GOSTA (2000): Fundamentos sociales de las economías postindustriales, Barcelona: Ariel.

ESPING-ANDERSEN, GOSTA y KORPI, WALTER (1991): "La política social como política de clase en el capitalismo de posguerra: Escandinavia, Austria y Alemania" en J.H. Goldthorpe (Ed.), Orden y conflicto en el capitalismo contemporaneo, Madrid: Ministerio de Trabajo y Seguridad Social: 253-92.

EVANS, PETER B., RUESCHEMEYER, DIETRICH y SKOCPOL, THEDA (1985): Bringing the state back in, Cambridge: Cambridge University Press.

FERNÁNDEZ, JUAN ANTONIO y TOBÍO, CONSTANZA (2005): "Conciliar las responsabilidades familiares y laborales: políticas y prácticas sociales", Madrid: Fundación Alternativas.

FERRERA, MAURIZIO y HEMERIJCK, ANTON (2003): "Recalibrating Europe's Welfare Regimes" en J. Zeitlin y D.M. Drubek (Ed.), Governing Work and Welfare in the New Economy: European and American Experiments, Oxford: Oxford University Press: 88-128.

GONZÁLEZ, MARÍA JOSÉ (2004): "La escolarización de la primera infancia en España: desequilibrios territoriales y socioeconómicos en el acceso a los servicios" en V. Navarro (Ed.), El Estado del Bienestar en España, Madrid: Tecnos: 291-312.

GORNICK, JANET C. y MEYERS, MARCIA K. (2009): "Institutions that Support Gender Equality in Parenthood and Employment" en J.C. Gornick y M.K. Meyers (Ed.), Gender Equality. Transforming Family Divisions of Labor, New York: Verso: 3-66.

GUILLÉN, ANA MARTA (2000): La construcción política del sistema sanitario español: de la postguerra a la democracia, Madrid: Ex Libris.

GUILLÉN, ANA MARTA y ÁLVAREZ, SANTIAGO (2004): "The EU's impact on the Spanish welfare state: the role of cognitive Europeanization", Journal of European Social Policy, 14 (3): 285-99.

GUILLÉN, ANA MARTA y PETMESIDOU, MARÍA (2008): "The publicprivate mix in Southern Europe: What changed in the last decade?" en M. SeeleibKaiser (Ed.), Welfare State Transformations. Comparative Perspectives, Basingstoke: Macmillan: 56-78.

HOBSON, BARBARA y LINDHOM, MARIKA (1997): "Collective Identities, Women's Power Resources, and the Making of Welfare States", Theory and Society, 26 (4): 475-508. 
HUBER, EVELYN y STEPHENS, JOHN D. (2001): Development and crisis of the Welfare State: parties and policies in global markets, Chicago: The University of Chicago Press.

JENSEN, CARSTEN (2009): "Institutions and the politics of childcare services", Journal of European Social Policy, 19 (1): 7-18.

KORPI, WALTER (1983): The democratic class struggle, London: Routledge \& Kegan Paul.

KORPI, WALTER (2000): "Faces of Inequality: Gender, Class, and Patterns of Inequalities in Different Types of Welfare States", Social Politics: International Studies in Gender, State \& Society, 7 (2): 127-91.

KORPI, WALTER (2006): "Power Resources and Employer-Centered Approaches in Explanations of Welfare States and Varieties of Capitalism: Protagonists, Consenters, and Antagonists", World Politics, 58 (2): 167-2006.

KORPI, WALTER y PALME, JOAKIM (2003): "New Politics and Class Politics in the Context of Austerity and Globalization: Welfare State Regress in 18 Countries, 1975-95", American Political Science Review, 97 (3): 425-46.

LEÓN, MARGARITA (2007): "Speeding up or holding back? Institutional factors in the development of childcare provision in Spain ", European Societies, 9 (3): 315-37.

LEÓN, MARGARITA (2011): "The Quest for Gender Equality" en A.M. Guillén y M. Léon (Ed.), The Spanish Welfare State in European Context, Farnham: Ashgate: 59-74.

LEVY, JONAH D. (1999): "Vice into Virtue? Progressive Politics and Welfare Reform in Continental Europe", Politics \& Society, 27 (2): 239-73.

MACINNES, JOHN (2006): "Work-Life Balance in Europe: A Response to the Baby Bust or Reward for the Baby Boomers?", European Societies, 8 (2): 223-49.

MÄTZKE, MARGITTA y OSTNER, ILONA (2010): "Introduction: Change and continuity in recent family policies", Journal of European Social Policy, 20 (5): 387-398.

MAHON, RIANNE (1997): "Child Care in Canada and Sweden: Policy and Politics", Social Politics, Fall: 382-418.

MARTÍNEZ, MARÍA (2011): "El movimiento feminista en la España contemporánea. Apuntes de una tesis en proceso de construcción", $V$ Jornadas de Estudios de Género en las Universidades Valencianas, Valencia.

MEYER, TRAUTE (2005): "Political actors and the modernisation of care policies in Britain and Germany" en B. Pfau-Effinger y B. Geissler (Ed.), Care and Social Integration in European Societies, Bristol: Policy Press: 281-305.

MORENO FUENTES, FRANCISCO JAVIER (2009): "Del sistema sanitario de la Seguridad Social al Sistema Nacional de Salud descentralizado" en L. Moreno (Ed.), Reformas de las políticas de bienestar en España, Madrid: Siglo XXI: 101-35.

MORENO, LUIS (2000): Ciudadanos precarios. La "última red" de protección social, Barcelona: Ariel. 
MORENO, LUIS (2009): "Nursopob: Presentación, contexto del bienestar y nuevos riesgos sociales" en L. Moreno (Ed.), Reformas de las politicas del bienestar en España, Madrid: Siglo XXI: 1-34.

MYLES, JOHN y PIERSON, PAUL (2001): "The Comparative Political Economy of Pension Reform" en P. Pierson (Ed.), The New Politics of the Welfare State, Oxford: Oxford University Press: 305-33.

NAUMANN, INGELA (2005): "Child care and feminism in West Germany and Sweden in the 1960 and 1970", Journal of European Social Policy, 15 (1): 47-63.

O'CONNOR, JULIA S. (1993): "Gender, class and citizenship in the comparative analysis of welfare state regimes: theoretical and methodological issues", British Journal of Sociology, 44 (3): 501-18.

O'CONNOR, JULIA S. (1998): "Gender, Class and Citizenship in the Comparative Analysis of Welfare State Regimes" en J.S. O'Connor y G.M. Olsen (Ed.), Power resources theory and the welfare state, a critical approach., Toronto: University of Toronto Press: 209-49.

OLIVER, REBECCA J. y MÄTZKE, MARGITTE (2014): "Childcare Expansion in Conservative Welfare States: Policy Legacies and the Politics of Decentralized Implementation in Germany and Italy", Social Politics, 21 (2): 167-193.

ORLOFF, ANN SHOLA (1993): "Gender and the Social Rights of Citizenship: The Comparative Analysis of Gender Relations and Welfare States", American Sociological Review, 58 (3): 303-28.

PAPADOPOULOS, THEO (2005): "The Recommodification of European Labour: Theoretical and Empirical Explorations", Working Paper Series, Bath: ERI, The European Research Institute.

PETERSON, ELIN (2007): "El género en los marcos interpretativos sobre la "conciliación de la vida familiar y laboral"” en M. Bustelo y E. Lombardo (Ed.), Políticas de Igualdad en España y en Europa. Afinando la mirada, Madrid: Cátedra: 37-66.

PETERSON, ELIN (2011): Beyond the "women-friendly" welfare state. Framing gender inequality as a policy problem in Spanish and Swedish politics of care, Tesis doctoral Universidad Complutense de Madrid, Madrid.

PIERSON, PAUL (1996): "The New Politics of the Welfare State", World Politics, 48 (2): 143-79.

PIERSON, PAUL (2001a): "Post-Industrial Pressures on the Mature Welfare States" en P. Pierson (Ed.), The New Politics of the Welfare State, Oxford: Oxford University Press: 80-104.

PIERSON, PAUL (2001b): "Coping with Permanent Austerity: Welfare State Restructuring in Affluent Democracies" en P. Pierson (Ed.), The New Politics of the Welfare State, Oxford: Oxford University Press: 410-57.

PLANTENGA, JANNEKE y RÉMERY, CHANTAL (2005): Reconciliation of Work and Private Life: A Comparative Review of Thirty European Countries. Report for European Commission, Bruselas: European Commission. 
RAMOS, JUAN ANTONIO y DEL PINO, ELOÍSA (2009): "Un análisis político del cambio en el sistema de pensiones en España" en L. Moreno (Ed.), Reformas de las políticas de bienestar en España, Madrid: Siglo XXI: 67-100.

RAUCH, DIETMAR (2005): "Institutional Fragmentation and Scandinavian Childcare Variations", Journal of Public Policy, 25 (3): 367-94.

RHODES, MARTIN (2001): "The political economy of social pacts: 'Competitive corporativism' and the European welfare reform" en P. Pierson (Ed.), The new politics of the welfare state, Oxford: Oxford University Press: 165-96

RIEGER, ELMAR y LEIBFRIED, STEPHAN (2003): Limits to Globalization: Welfare States and the World Economy, Malden: Polity Press.

RODRÍGUEZ CABRERO, GREGORIO (2006): "La reforma permanente del sistema de pensiones" en G. Rodríguez Cabrero, et al. (Ed.), Actores Sociales y Reformas del Bienestar, Madrid: Centro Superior de Investigaciones Científicas: 28-52.

SAINSBURY, DIANE (1999): Gender and Welfare State Regimes, Oxford Oxford University Press.

SALIDO, OLGA y MORENO, LUIS (2009): "Familia y género" en L. Moreno (Ed.), Reformas de las políticas del bienestar en España, Madrid: Siglo XXI: 281-308.

SEELEIB-KAISER, MARTIN (2008): "Welfare State Transformations in Comparative Perspective: Shifting Boundaries of 'Public' and 'Private' Social Policy?" en M. Seeleib-Kaiser (Ed.), Welfare State Transformations. Comparative Perspectives, Basingstoke: Palgrave Macmillan: 1-13.

SERRANO, AMPARO y MAGNUSSON, LARS (Ed.) (2007): Reshaping Welfare States and Activation Regimes in Europe, Bruselas: P.I.E. Peter Lang.

SKOCPOL, THEDA (1992): Protecting Soldiers and Mothers: The Political Origins of Social Policy in the United States, Cambridge: harvard University Press.

STREECK, WOLFGANG y THELEN, KATHLEEN (Ed.) (2005): Beyond Continuity. Institutional Change in Advanced Political Economies, Oxford: Oxford University Press.

TAYLOR-GOOBY, PETER (2004): "New Risks and Social Change" en P. Taylor-Gooby (Ed.), New Risks, New Welfare. The Transformation of the European Welfare State, Oxford Oxford University Press: 1-28.

TOBÍO, CONSTANZA (2005): Madres que trabajan: Dilemas y Estrategias, Madrid: Cátedra.

UGT (2008): 100 años trabajando por la igualdad. Informe 8 de marzo 2008, Madrid: Departamento General de la Mujer UGT, Secretaría para la Igualdad.

UNICEF (2014): La infancia en España 2014. El valor social de los niños: Hacia un Pacto de Estado por la Infancia, Madrid: Unicef.

VALIENTE, CELIA (1996): "El feminismo institucional en España: El Instituto de la Mujer, 1983-1994", Revista Internacional de Sociología, 13 (Ener-Abril): 163-204.

VALIENTE, CELIA (2000): "Reconciliation policies in Spain" en L. Hantrais (Ed.), Gendered policies in Europe. Reconciling employment and family life, Basingstoke: Macmillan: 143-59. 
VALIENTE, CELIA (2005): "Combating violence against women" en M. Threlfall, C. Cousins y C. Valiente (Ed.), Gendering Spanish Democracy, Londres: Routledge: 101-24.

VERGE, TÀNIA (2006): "Mujer y partidos políticos en España: las estrategias de los partidos y su impacto institucional, 1978-2004", Revista Española de Investigaciones Sociológicas, 115 (Julio-Septiembre): 165-96.

WILLIAMS, FIONA (2002): "The presence of feminism in the future of welfare", Economy and Society, 31 (4): 502-19.
WORLD
SOCIAL
SURVEY

(2014):

http://www.worldvaluessurvey.org/WVSOnline.jsp. Fecha de consulta: 02/07/2014. 